\title{
Beneficial effects of linagliptin in cell culture model of Parkinson's disease
}

\author{
Ufuk Okkay® ${ }^{\oplus}$ Irmak Ferah Okkay ${ }^{2} \odot$
}

${ }^{1}$ Department of Medical Pharmacology, Atatürk University, Faculty of Medicine, Erzurum, Turkey

${ }^{2}$ Department of Pharmacology, Atatürk University, Faculty of Pharmacy, Erzurum, Turkey

\section{ABSTRACT}

Objectives: We aimed to investigate the neuroprotective effects of linagliptin in an in vitro 6-hydroxydopamine (6-OHDA) Parkinson's disease model.

Methods: 6-OHDA $(200 \mu \mathrm{M})$ were administered to the SH-SY5Y cells for $24 \mathrm{~h}$ to induce Parkinson's disease model in vitro. Cells were treated with linagliptin (1, 10, 50 and $100 \mathrm{nM}) 30$ minutes before 6-OHDA administration. Cell viability was examined by 3-[4,5-dimethylthiazole-2-yl]-2,5-diphenyltetrazolium bromide (MTT) method and lactate dehydrogenase (LDH) analysis. Superoxide dismutase (SOD), catalase (CAT), malondialdehyde (MDA) and reactive oxygen species (ROS) analyses were conducted to assess oxidative stress. Apoptosis was evaluated with Caspase-3 mRNA expression levels.

Results: It was observed that 6-OHDA elevated LDH levels and cell death. Oxidative stress was exaggerated with increased ROS and MDA levels and substantially apoptosis was proven with increased Caspase-3 levels in SH-SY5Y cells. Pretreatment with linagliptin alleviated oxidative stress and apoptosis.

Conclusions: Given its neuroprotective role as well as its effects on oxidative stress and apoptosis, linagliptin may be a drug candidate in Parkinson's disease.

Keywords: 6-OHDA, DPP-4 inhibitor, linagliptin, Parkinson's disease, SH-SY5Y cells

$\mathrm{P}$ arkinson's disease (PD) is a progressive and common neurodegenerative disease [1]. Characteristic feature of PD is progressive death of neuronal populations, primarily dopaminergic neurons [1]. Even though neuropathological processes of PD are well defined, its etiology is still largely undefined [2]. Furthermore, multiple mechanisms have been investigated as triggers of neuron loss in PD, including oxidative stress, mitochondrial dysfunction, apoptosis, and inflammation $[3,4]$. PD patient's key symptoms are tremor, bradykinesia, stiffness. Also, postural instability, behavioral and cognitive problems such as dementia, depression, anxiety, and sleep disturbances also occur in the late stages of PD [5].

Levodopa and/or dopamine agonists are used as the first choice in the treatment of PD, and the treatment has remained symptomatic [6]. These treatments are capable of slowing the progression of PD and most of them only relieving symptoms, and after a certain period most Parkinson's patients suffer from side effects such as motor and non-motor fluctuations and dyskinesia [7].

Linagliptin (LNG) is a dipeptidyl peptidase-4 (DPP-4) inhibitor which can be chosen for the type 2 
diabetes management. Several studies in recent years have demonstrated the strong neuroprotective properties of LNG in various neurodegenerative disorders, like Alzheimer's disease, dementia and stroke $[8,9]$. These effects are based on the antioxidant and anti-inflammatory features of LNG, as well as its capability to modify the crucial neurotransmitters' activity. It is also well known that glucagon-like peptide-1 (GLP1) exerts neuroprotective activity by attenuating neuroinflammation $[10,11]$.

6-hydroxydopamine (6-OHDA) is a neurotoxicant that has been extensively utilized to induce in vivo and in vitro experimental PD models. Together with other free radicals, it produces $\mathrm{H}_{2} \mathrm{O}_{2}, \mathrm{O} 2$ - and $\mathrm{OH}$ radicals, which determine mitochondrial membrane permeability loss and consequently results in oxidative stress $[12,13]$.

On this basis, this research was planned to assess the beneficial effects of LNG in 6-OHDA-induced in vitro PD model.

\section{METHODS}

\section{Cell Culture}

SH-SY5Y cells were incubated with $10 \%$ FBS and antibiotic solution in DMEM. The flask was cultivated at $37{ }^{\circ} \mathrm{C}$ with $5 \% \mathrm{CO} 2$. Then, $0.5 \times 10^{4}$ cells were seeded into 96 well-plates. To form PD in cell line, 6-OHDA $(200 \mu \mathrm{M})$ was administered to each well for one day. Firstly SH-SY5Y cells were treated with $\mathrm{LNG}(1,10,50$ and $100 \mathrm{nM})$ and then thirty minutes later 6-OHDA was administered to each well [1416].

\section{MTT Analysis}

In order to evaluate the cell viability, 3-[4,5-dimethylthiazole-2-yl]-2,5-diphenyltetrazolium bromide (MTT) method was utilized. $20 \mu 1$ MTT solution (Sigma-Aldrich) was added to each well. After four hours supernatants were exchanged with $150 \mu \mathrm{m}$ DMSO and the absorbance was measured at $490 \mathrm{~nm}$.

\section{LDH Analysis}

Lactate dehydrogenase (LDH) is an intracellular enzyme which reflects cytotoxicity. LDH leakages from cells when membrane integrity is disrupted. LDH activity were assessed by an LDH assay kit (Elab- science, US). Absorbance was calculated at $450 \mathrm{~nm}$.

\section{Oxidative Stress Markers}

Superoxide dismutase (SOD), catalase (CAT), malondialdehyde (MDA) and reactive oxygen species (ROS) were analyzed by ELISA kits (Elabscience, US) as described before [17]. Levels of ROS were measured using ELISA kit (LSBio, United states) and the optical density was assessed at $450 \mathrm{~nm}$.

\section{Molecular Analysis}

mRNA extraction and cDNA synthesis were carried out as previously described with RNeasy easy kit (Qiagen, Germany) Real-time PCR analysis also was carried out as previously described [18]. Relative mRNA caspase-3 expression levels were determined by Rotor-Gene Q (QIAGEN). $\beta$-actin was utilized as the reference gene. The target gene expression levels were compared with the housekeeping gene $\beta$-actin. The sequences of specific primers were as follows: Caspase-3: forward, 5'-TTTTCAGTCCGGGGACAAAC3', reverse, 5'-GGGCAGCCGAGAATAACAAT-3', $\beta$-actin: forward, 5'- CAAGGTGGGTGTCTTTCCTG-3', reverse, 5'-GATCCACACGGAGTACTTGC-3'. The obtained results were analyzed as fold changes using the $2^{-\Delta \Delta \mathrm{Ct}}$ method.

\section{Statistical Analysis}

All data were analyzed with one-way analysis of variance (ANOVA) followed by the Tukey post hoc test (IBM SPSS 21.0) $p<0.05$ assumed meaningful. Results are mean \pm SD.

\section{RESULTS}

\section{MTT and LDH Analyses}

6-OHDA lead to marked reduction of cell viability in the MTT assay and increased LDH leakage. Also, LNG increased the cell proliferation and our findings proved that LNG markedly increased viable cell ratio. Additionally, LNG significantly reduced LDH levels (Fig. 1).

\section{Oxidative Stress Results}

SOD and CAT levels were markedly decreased in 6-OHDA group while MDA and ROS levels significantly increased in comparison to control group. Activities of SOD and CAT were significantly increased 
MTT

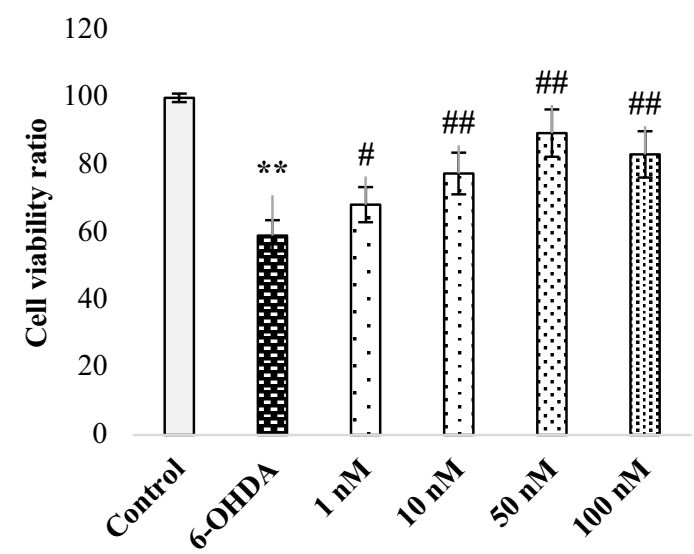

LDH

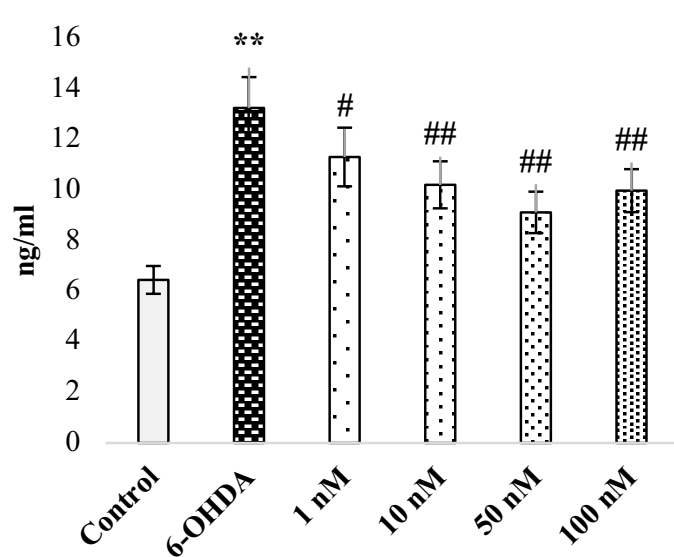

Fig 1. Cell viability test results. Results are mean \pm SD. $* * p<0.001$ versus control, $\# p<0.05$ versus $6-0$ HDA, \#\# $p<0.001$ versus 6-OHDA.

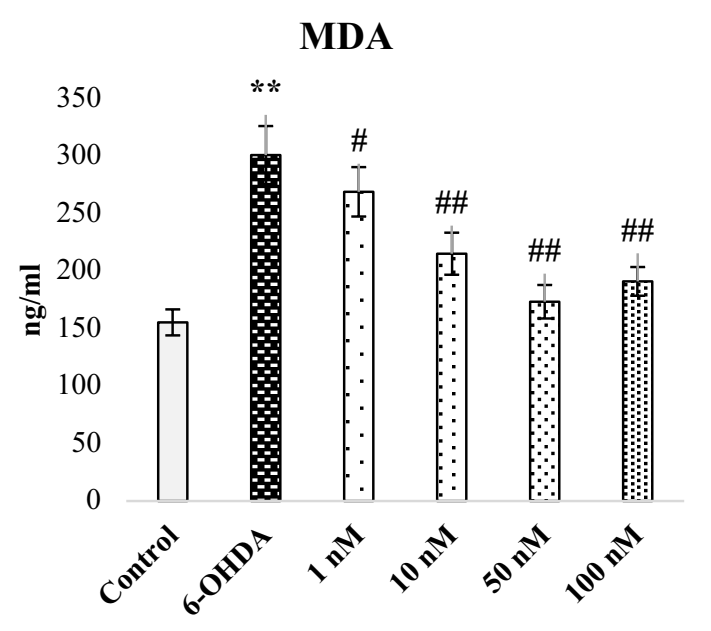

CAT

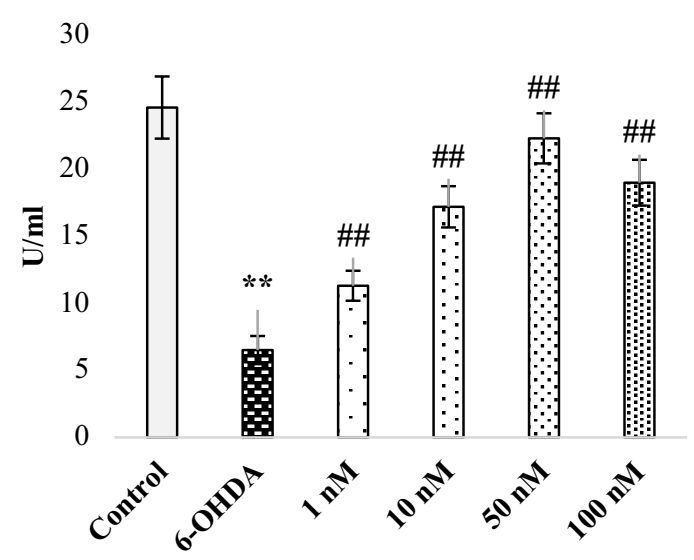

SOD

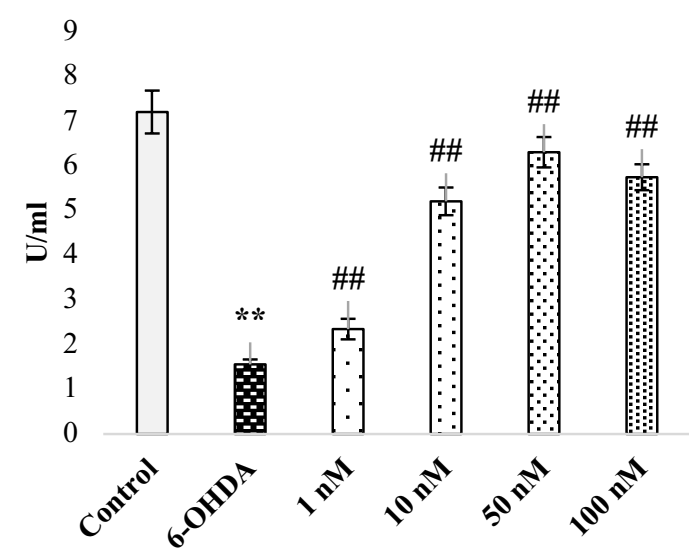

ROS

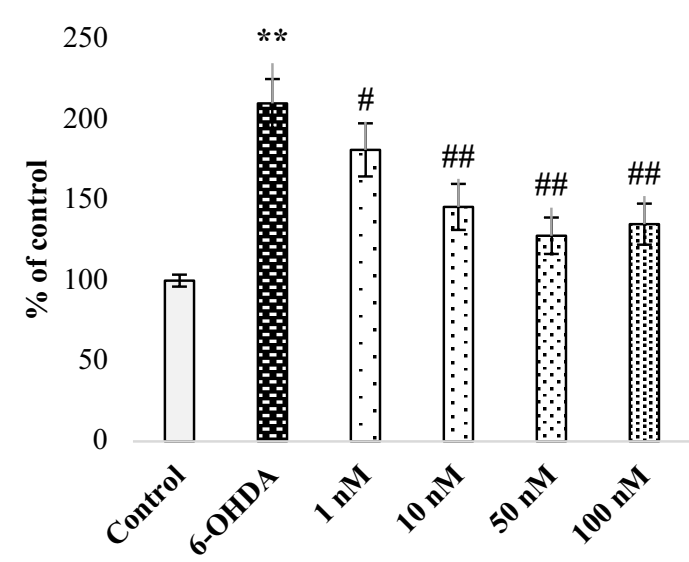

Fig 2. Oxidative stress results. Results are mean \pm SD. ${ }^{* *} p<0.001$ versus control, \#p $<0.05$ versus 6-OHDA, \#\#p $<0.001$ versus 6-OHDA.

in LNG groups, whereas MDA and ROS concentrations were markedly decreased in comparison with 6OHDA group (Fig. 2).
mRNA Expressions of Caspase-3

The mRNA expression of Caspase- 3 was markedly increased in the 6-OHDA group. LNG ad- 
ministration significantly reduced caspase- 3 expression in comparison with 6-OHDA group (Fig. 3).

\section{DISCUSSION}

In this research, the neuroprotective effects of LNG against 6-OHDA neurotoxicity in SH-SY5Y cells was examined for the first time.

In this study, we observed that 6-OHDA significantly reduced SH-SY5Y cell viability, and treatment with LNG increased cell viability. In addition to our observations of cell viability, we also demonstrated a significant reduction of apoptosis in LNG groups. GLP-1, an incretin hormone which has been proven to be effective in neurodegenerative disorders like Alzheimer's disease and GLP-1 is immediately de-activated by DPP-4 enzyme. Thus, inhibition of DPP-4 is used for elevation of GLP-1. There are studies confirming that GLP-1 analogs improve neurodegeneration by reversing cognitive deficits in neurodegenerative disorders like Alzheimer's disease $[19,20]$. Also, DPP-4 inhibition is supposed to exert neuroprotective effects by elevating GLP-1 levels in circulation. De-activation of DPP-4 has been shown to modulate the levels of glucose-dependent insulinotropic polypeptide, neuropeptide Y, brain natriuretic peptide, and stromal-derived factor-1, which have neuroprotective effects [21-23]. These data re-

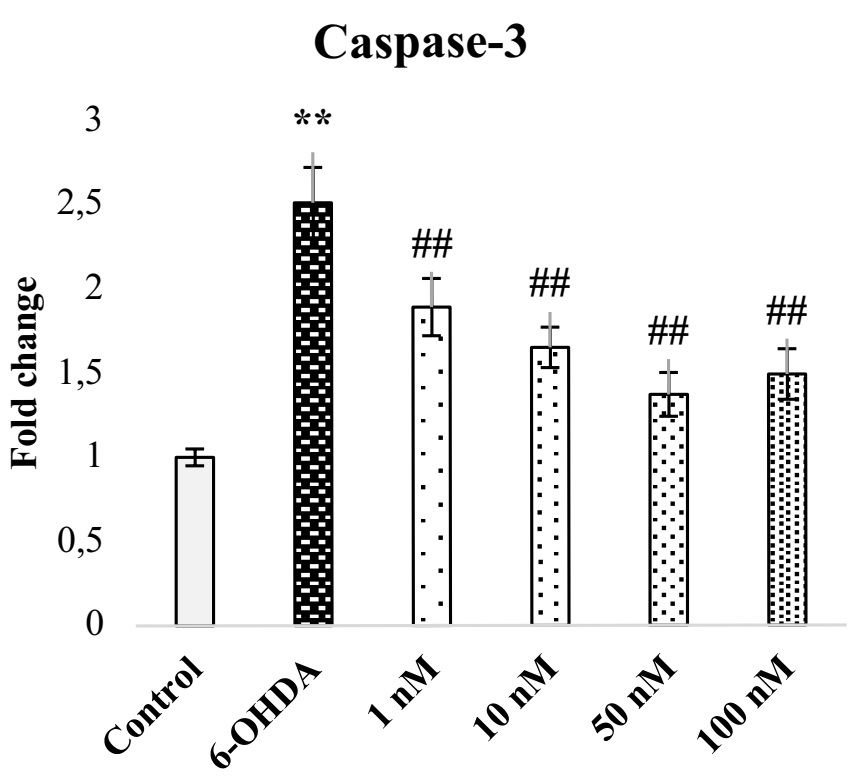

Fig 3. Relative expression of Caspase-3. Results are mean \pm SD. ${ }^{* *} p<0.001$ versus control, \#\#p<0.001 versus 6-OHDA. vealed that by inhibiting the DPP-4 enzyme, neuroprotective effects can be obtained in neurodegenerative diseases such as PD.

Oxidative damage is known to take part in pathological processes associated with neurodegenerative disorders like PD [24]. Previously, it has been proven that overproduction of ROS in PD can destroy neuronal cell function, cause oxidative DNA damage, disrupt the respiratory chain, and disrupt mitochondrial DNA mutations in the brain of patients with PD [25]. Inhibiting ROS formation and increasing antioxidant enzyme activity are valuable strategies to reduce oxidative stress. In this report, LNG decreased the ROS and MDA concentrations and elevated SOD and CAT activity. Besides, similar to our results, LNG has been reported to have a broad antioxidant effect by reducing ROS production and increasing the activities of antioxidant enzymes [16]. Also, it has been previously reported that LNG exerts an indirect antioxidant effect by increasing the level of circulating GLP-1, which has strong antioxidant, anti-inflammatory and neuroprotective effects in the central nervous system [9].

A substantial amount of evidence indicates that 6OHDA also induces apoptosis through caspase activation following oxidative stress with excessive ROS increase. Caspase- 3 is a substantial component of the cysteine protease class concerned with in the mitochondrial apoptotic pathway [26]. We found that LNG down-regulated caspase-3 expression and showed antiapoptotic effects, confirming previous studies. Previously it has been reported that LNG has a marked neuroprotective, anti-apoptotic and cognitive improving properties in in-vitro cerebral ischemia model [27].

\section{CONCLUSION}

In the light of all these data, LNG may be a promising agent in Parkinson's disease with its anti-oxidant and anti-apoptotic properties.

\section{Authors' Contribution}

Study Conception: IFO, UO; Study Design: IFO, UO; Supervision: IFO, UO; Funding: N/A; Materials: N/A; Data Collection and/or Processing: IFO, UO; Statistical Analysis and/or Data Interpretation: IFO, UO; Literature Review: IFO, UO; Manuscript Preparation: IFO and Critical Review: IFO, UO. 


\section{Conflict of interest}

The authors disclosed no conflict of interest during the preparation or publication of this manuscript.

\section{Financing \\ The authors disclosed that they did not receive any grant during conduction or writing of this study.}

\section{REFERENCES}

1. Chahine LM, Amara AW, Videnovic A. A systematic review of the literature on disorders of sleep and wakefulness in Parkinson's disease from 2005 to 2015. Sleep Med Rev 2017;35:33-50. 2. Olanow CW, Stern MB. Parkinson's disease: unresolved issues. Ann Neurol 2008;64 Suppl 2:S1-2.

3. Hirsch EC, Hunot S, Faucheux B, Agid Y, Mizuno Y, Mochizuki H, et al. Dopaminergic neurons degenerate by apoptosis in Parkinson's disease. Mov Disord 1999;14:383-5.

4. Miller RL, James-Kracke M, Sun GY, Sun AY. Oxidative and inflammatory pathways in Parkinson's disease. Neurochem Res 2009;34:55-65.

5. Reeve A, Simcox E, Turnbull D. Ageing and Parkinson's disease: why is advancing age the biggest risk factor? Ageing Res Rev 2014;14:19-30.

6. Foltynie T, Kahan J. Parkinson's disease: an update on pathogenesis and treatment. J Neurol 2013;260:1433-40.

7. Ossig C, Reichmann H. Treatment of Parkinson's disease in the advanced stage. J Neural Transm (Vienna) 2013;120:523-9.

8. Kosaraju J, Holsinger RMD, Guo L, Tam KY. Linagliptin, a Dipeptidyl peptidase-4 inhibitor, mitigates cognitive deficits and pathology in the 3xTg-AD mouse model of Alzheimer's disease. Mol Neurobiol 2017;54:6074-84.

9. Darsalia V, Ortsater H, Olverling A, Darlof E, Wolbert P, Nystrom T, et al. The DPP-4 inhibitor linagliptin counteracts stroke in the normal and diabetic mouse brain: a comparison with glimepiride. Diabetes 2013;62:1289-96.

10. McClean PL, Parthsarathy V, Faivre E, Holscher C. The diabetes drug liraglutide prevents degenerative processes in a mouse model of Alzheimer's disease. J Neurosci 2011;31:6587-94.

11. Duffy AM, Holscher C. The incretin analogue D-Ala2GIP reduces plaque load, astrogliosis and oxidative stress in an APP/PS1 mouse model of Alzheimer's disease. Neuroscience 2013;228:294-300.

12. Xicoy H, Wieringa B, Martens GJ. The SH-SY5Y cell line in Parkinson's disease research: a systematic review. Mol Neurodegener 2017;12:10.

13. Hernandez-Baltazar D, Zavala-Flores LM, Villanueva-Olivo A. The 6-hydroxydopamine model and parkinsonian pathophysiology: novel findings in an older model. Neurologia 2017;32:533-9.

14. Li H, Zhang J, Lin L, Xu L. Vascular protection of DPP-4 in- hibitors in retinal endothelial cells in in vitro culture. Int Immunopharmacol 2019;66:162-8.

15. Shi S, Kanasaki K, Koya D. Linagliptin but not sitagliptin inhibited transforming growth factor-beta2-induced endothelial DPP-4 activity and the endothelial-mesenchymal transition. Biochem Biophys Res Commun 2016;471:184-90.

16. Nakamura Y, Inagaki M, Tsuji M, Gocho T, Handa K, Hasegawa $\mathrm{H}$, et al. Linagliptin has wide-ranging anti-inflammatory points of action in human umbilical vein endothelial cells. Jpn Clin Med 2016;7:27-32.

17. Okkay U, Ferah Okkay I, Aydin IC, Bayram C, Ertugrul MS, Gezer A, et al. Effects of Achillea millefolium on cisplatin induced ocular toxicity: an experimental study. Cutan Ocul Toxicol 2021;40:214-20.

18. Okkay U, Ferah Okkay I, Cicek B, Aydin IC, Ertugrul MS, Bayram C, et al. Achillea millefolium alleviates testicular damage in paclitaxel-intoxicated rats via attenuation of testicular oxidoinflammatory stress and apoptotic responses. Andrologia 2021;53:e14028.

19. Calsolaro V, Edison P. Novel GLP-1 (Glucagon-Like Peptide-1) analogues and insulin in the treatment for Alzheimer's disease and other neurodegenerative diseases. CNS Drugs 2015;29:1023-39.

20. Holscher C. Incretin analogues that have been developed to treat type 2 diabetes hold promise as a novel treatment strategy for Alzheimer's disease. Recent Pat CNS Drug Discov 2010;5:109-17.

21. Shannon RP. DPP-4 inhibition and neuroprotection: do mechanisms matter? Diabetes 2013;62:1029-31.

22. Ji C, Xue GF, Lijun C, Feng P, Li D, Li L, et al. A novel dual GLP-1 and GIP receptor agonist is neuroprotective in the MPTP mouse model of Parkinson's disease by increasing expression of BNDF. Brain Res 2016;1634:1-11.

23. Spencer B, Potkar R, Metcalf J, Thrin I, Adame A, Rockenstein E, et al. Systemic central nervous system (CNS)-targeted delivery of neuropeptide Y (NPY) reduces neurodegeneration and increases neural precursor cell proliferation in a mouse model of Alzheimer disease. J Biol Chem 2016;291:1905-20.

24. Xu Z, Yang D, Huang X, Huang H. Astragaloside IV protects 6-hydroxydopamine-induced SH-SY5Y cell model of Parkinson's disease via activating the JAK2/STAT3 pathway. Front Neurosci 2021;15:631501.

25. Medeiros MS, Schumacher-Schuh A, Cardoso AM, Bochi GV, Baldissarelli J, Kegler A, et al. Iron and oxidative stress in Parkinson's disease: an observational study of injury biomarkers. PLoS One 2016;11:e0146129.

26. Ghobrial IM, Witzig TE, Adjei AA. Targeting apoptosis pathways in cancer therapy. CA Cancer J Clin 2005;55:178-94.

27. El-Deeb OS, Soliman GM, Elesawy RO. Linagliptin, the dipeptidyl peptidase-4 enzyme inhibitor, lessens CHOP and GRP78 biomarkers levels in cisplatin-induced neurobehavioral deficits: A possible restorative gateway. J Biochem Mol Toxicol 2020:e22541. 